\title{
Multispectral Image Processing System Developed in CARTOMORPH Software-NDVI Module
}

\section{Tiago Oyan Aguiar, Samara Calçado de Azevedo, Miriam Maria Pedrosa, Guilherme Pina Cardim, Erivaldo Antonio da Silva}

Department of Cartography, São Paulo State University (UNESP), Presidente Prudente, Brazil

Email: tiago_oyan@hotmail.com, samara_calcado@hotmail.com, miriammp@hotmail.com, gpcardim@hotmail.com, silva.erialdo@gmail.com

How to cite this paper: Aguiar, T.O., de Azevedo, S.C., Pedrosa, M.M., Cardim, G.P. and da Silva, E.A. (2018) Multispectral Image Processing System Developed in CARTOMORPH Software-NDVI Module. Advances in Remote Sensing, 7, 91-100. https://doi.org/10.4236/ars.2018.72007

Received: April 3, 2018

Accepted: June 19, 2018

Published: June 22, 2018

Copyright $\odot 2018$ by authors and Scientific Research Publishing Inc. This work is licensed under the Creative Commons Attribution International License (CC BY 4.0).

http://creativecommons.org/licenses/by/4.0/

\begin{abstract}
In this paper, we have evaluated the multispectral module implemented in the CARTOMORPH software, which is a public domain software under development by the mathematical morphology research group at FCT/UNESP. The aim of CARTOMORPH is to provide an open software with feature extraction methods and a library that contains a variety of implemented code (functions) that can be easily operated by users through graphical interface. The multispectral image processing system has been developed to allow feature discrimination by operations between bands from Remote Sensing images. The normalized difference vegetation index (NDVI) was selected to be implemented on CARTOMORPH because of its acknowledged performance for monitoring of vegetation and cartographic applications. The experiments were applied to multispectral bands from Quick Bird image and the results were compared with those provided by the SPRING and ENVI software. Former is a Brazilian free software, developed by National Institute for Space Research-INPE and dedicated to image processing and Geographic Information Systems (GIS) analysis, as well as ENVI, which is a traditional remote sensing image analysis system. The results prove that the implementation of this module is correct, allowing potential usage in the field of cartography and for environmental applications.
\end{abstract}

\section{Keywords}

NDVI, CARTOMORPH, Quick Bird, Remote Sensing

\section{Introduction}

The society's demand for up-to-date surface information has been steadily in- 
creasing and has a direct contribution to continuous technological progress in the production of maps. In the Brazilian context, as well as in other countries of continental scale, reliable alternatives to deal with cartographic updating are needed in order to obtain consistent and economically viable cartographic databases. One of the possible solutions employed to solve this ongoing problem has been data integration from remote sensing (RS) with digital image processing (DIP) techniques [1].

RS imagery are timely and cost-effective data which plays a significant role in a wide range of real-world applications, such as natural disasters assessment [2], vegetation monitoring [3] and climate change understanding [4]. The developments of sensors and imagery have required improvements in computer software and hardware to handle with data complexity. Besides the increased availability of spatial details, new challenges were imposed to the traditional RS methods which are a core research topic to be pursued [5].

For this reason, the Mathematical Morphology (MM) research group at the FCT/UNESP, Presidente Prudente has developed a software named CARTOMORPH. CARTOMORPH is a public domain software and it is available with open source, allowing anyone to obtain efficient results for various applications within digital image processing [6].

One of the proposals of CARTOMORPH software is to establish functions that produce results consistent with commercials software currently available. Some routines and functions for features extraction based on morphological processing are already available in its library. However, most of these routines enable operations with individual monochromatic images. This can restrict many analysis techniques, since spectral properties permit that different types of target, such as vegetation species, soil, crop or rock types, can be distinguished from their spectral signatures on multispectral images [7].

The development of the CARTOMORPH multispectral module is clearly necessary to provide reliable identification of particular targets on remotely sensed images. This paper presents the results obtained in the processing module developed and implemented in CARTOMORPH. At this stage, NDVI spectral rationing was selected for deployment, because of its valuable contribution in monitoring vegetation and environmental studies of indicators of vegetation health or productivity [8] [9]. Additionally, the cartographic research field also takes advantage of the spectral ratio, since topographic effects and variations in scene illumination are reduced, and the resulting image better reflects real characteristics of the surface [7].

Regarding the importance of assessing the results from the multispectral module, we carried out a comparison with NDVI results obtained from SPRING, which is also a Brazilian free Geografical Information System (GIS) and image processing software developed by the National Institute for Space Research (INPE) and widely used in Brazilian context applications. In addition, ENVI was also used on the comparison carried out, since it is an important RS image anal- 
ysis system in the cartography context. The comparison of the outcomes allows information to be gained about the efficiency and agreement of both software's, in order to verify CARTOMORPH's performance and its ability to provide reliable results.

\section{Literature Review}

Operations between bands from RS images are simple spectral transformations of two or more bands, designed to enhance or to characterize targets present on the surface. The process consists of bringing up certain aspects of different Earth surface cover types based on the behaviour of its spectral reflectance. Image division or Spectral Rationing, for example, involves dividing two different spectral bands in order to enhance variations of the spectral reflectance curves and highlight suitable variations in the spectral responses of various surface targets [1].

One of the earliest known spectral ratios used in RS applications, being able to highlight dense vegetation cover, is called the vegetation index. Figure 1 presents the vegetation spectral reflectance curve to illustrate the practical concept of the index, where two more reflectance spectra (water and soil) are also illustrated. The differences between the curves can easily be seen, mainly due to slope between the red and the near-infrared bands, for example bands 3 (red) and 4 (near-infrared) in a Landsat ETM + image, as also stated on Figure 1.

In the vegetation spectrum, the main feature absorption is in the visible red waveband (centred on $650 \mathrm{~nm}$ ), and reflects strongly in the near infrared, where the wavelength from $760 \mathrm{~nm}$ to $900 \mathrm{~nm}$ is situated [10]. Chlorophyll pigments in the leaves, which are photosynthetically active, absorb incident energy, being indicative of healthy vegetation, and cause the absorption effect.

Therefore, the Normalized Difference Vegetation Index (NDVI) exploits this spectral vegetation behaviour, using the ratio between the near infrared and red band, as showed by Equation (1).

$$
N D V I=\frac{\rho_{\mathrm{NIR}}-\rho_{R}}{\rho_{N I R}+\rho_{R}}
$$

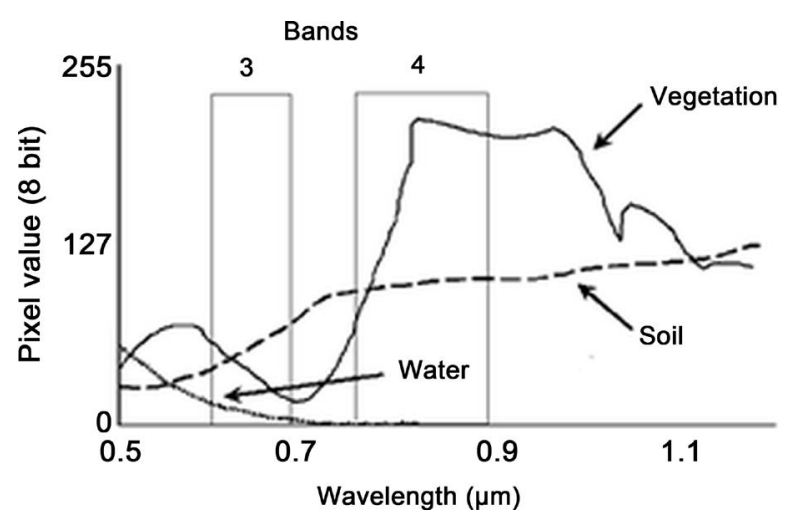

Figure 1. The reflectance curve from targets shows high slope between the red and near infrared bands (band 3 and 4 respectively) for vegetation and almost null gradient for water and soil. Adapted from [1]. 
Results obtained from NDVI is known to range between \pm 1 , where generally the higher pixels values are correlated with vegetation cover, density and vigour. Pixels from other targets, such as water, result in values less than or equal to 0 , while the soil gives values less than or equal to 0.5 . NDVI is computed as floating-point values, which can be resampled to integer values for display purposes, for example a monochromatic image on the range 0 - 255.

The ability of NDVI to highlight vegetation properties through remotely sensed images has been a key for assessing and managing natural resources. The main advantage is the capacity of RS offer systematic data over large areas, which goes beyond traditional methods [11]. Therefore, NDVI allows global vegetation studies and monitoring seasonal and inter-annual of vegetation phenological, and biophysical parameters, which are essential to better understanding the land cover and associated changes [12].

More recently, new indices based on NDVI has been created to overcome problems in dense forest canopies as well as seen new functionalities allowed by recent multispectral sensors [13]. Thus, the continuous development of various spectral indices for the rapid and accurate classification of land use and land cover imposes the development of algorithms and software, such CARTOMORPH, that are able to deal with this data.

\section{Study Area and Data Sources}

The experiments were carried out on QuickBird (QB) image of April 2009 from Maracaí city, located in São Paulo State. The multispectral imagery used in the application of NDVI was red (R) and near infrared (NIR) bands with $2.7 \mathrm{~m}$ of spatial resolution. Figure 2 shows the geographical location of the study site inside of Brazil, with false color composite RGB-432 to better illustrate existing vegetation in this area.

As can be seen in Figure 2, the study area contains few urban segments represented in cyan colour (such as streets, commercial and residential buildings), bare soil in green and small area of water body showed in dark green. The most of the land cover is occupied by vegetation as grassland and sugarcane cultivation, represented by several tones of red. The presence of dense vegetation can also be seen in dark red. The high spatial resolution of QB image enhances surrounding targets identification and, as we can see, the scene have no cloud contamination, which is essential to achieve better NDVI results.

\section{Methodology}

CARTOMORPH software, as mentioned above, is for public domain and developed by students at FCT/UNESP. It has been implemented in C++ in the NetBeans development platform. The methodology was aimed at NDVI implementation on CARTOMORPH software and validation of results using the test images. A priori, the algorithm was named as " $c m N D V P$ ', where the initials " $\mathrm{cm}$ " is an abbreviation of CARTOMORPH. 

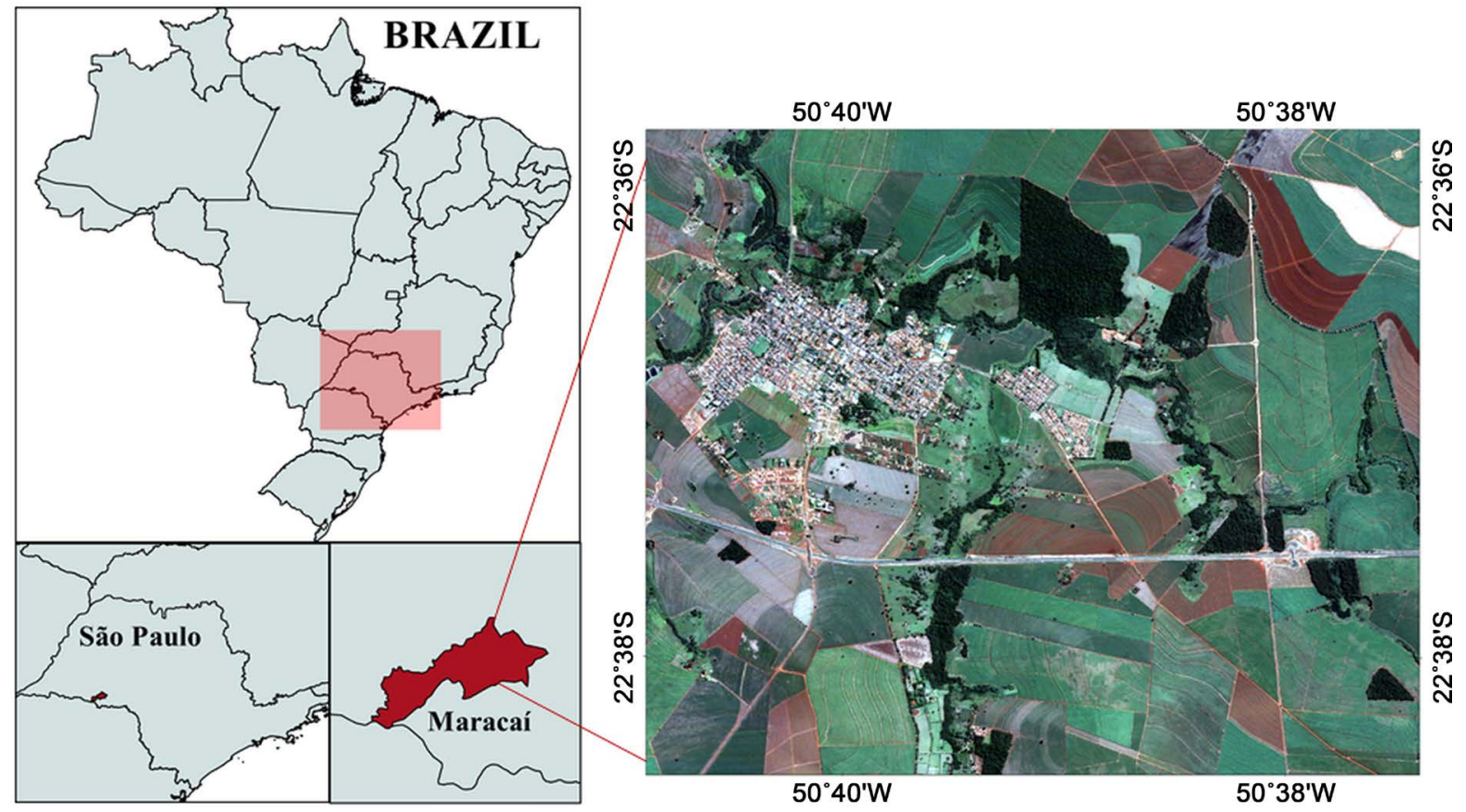

Figure 2. The geographical location of the study area and QB false color composite used to the application of NDVI.

In this study, we focused on verifying the correct implementation of $c m N D V I$. Thus, the experiments were carried out in digital values, and the dynamic range of the QB image was resampled to 8 bits, since CARTOMORPH is currently operating with this data.

The next step in the implementation, was the processing of the images by CARTOMORPH software. Depending on the applied operation, there may be loss of information when the new values exceed the range of digital numbers or result in fractional numbers. For example, an 8 bits image, after processing operation, for values that fall below 0 will be defined as 0 . On the other hand, any value greater than 255 is shifted to 255 , which is required to maintain the dynamic range purpose at 8 bits of radiometric resolution. Typically, in the band rationing process, a reducing of digital values happens to very small values. It is therefore necessary to consider parameters of gain (multipliers) and an offset (additive), as shown in Equation (2). Thus, the $c m N D V I$ algorithm was based and applied following Equation (2).

$$
c m N D V I=\text { Gain } * \frac{\rho_{\mathrm{NIR}}-\rho_{R}}{\rho_{N I R}+\rho_{R}}+\text { Offset }
$$

Finally, we performed a comparative analysis between results from $c m N D V I$ and the outcome of NDVI from SPRING and ENVI software. The last two software were used as a mathematical proof to verify whether the cmNDVI was implemented properly and to determine whether the results are coherent.

\section{Presentation and Results Analysis}

Multispectral RS data has enabled the development of different mathematical 
combinations between spectral bands to acquire information from images. Vegetation indices, as NDVI, is one example of success in this sense, and has contributed to several applications such as monitoring vegetation cover and assessing ecosystem changes [14]. Therefore, the implementation of $c m N D V I$ enables CARTOMORPH to be placed in the free image processing software context.

The results obtained with the $c m N D V I$ algorithm for the whole image of the study area are shown in Figure 3. In order to provide better visualization, the output values were scaled to the range 0 - 255 through gain and offset parameters, defined empirically as being 80 and 100, respectively. This setting applied resulted in a monochromatic image within the range $[20,180]$.

It can be seen from the monochromatic image, when compared with the original false color composite (Figure 2), that vegetation regions are represented by brighter grey pixels. This output was compatible, as discussed by [7], where vegetation regions are represented by higher values due to differences between bands. In the case of absence of vegetation, such as bare soil or water body regions, we found mid and darker grey pixels, as well as some areas prepared for planting.

Although it is possible to identify features such as road and urban regions in the resulting monochromatic image, we performed a transformation of the dynamic range into color scale image. Figure 4 represents this color map transformation, where we can clearly see targets that do not pose vegetation areas in purple and dense vegetation coverage in dark green. These labels are based on

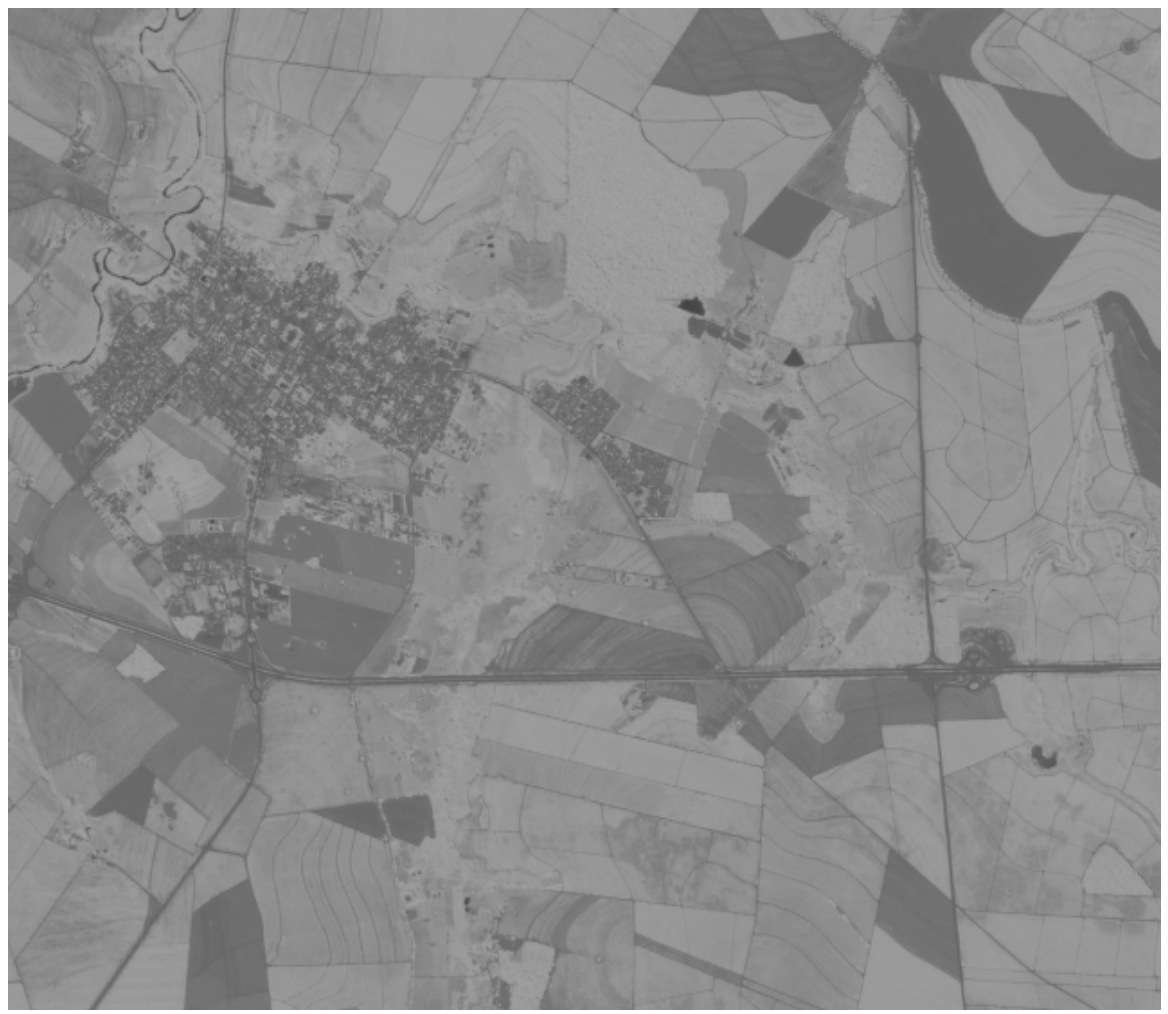

Figure 3. Monochromatic results achieved with cmNDVI for study area. 
the spectral characteristics of each pixel location.

The SPRING and ENVI software was used for comparison, as mentioned earlier, and the results found with NDVI were visually identical. In order to verify the results numerically, the morphological operator mmisequal from SDC Information Systems toolbox was used. This operator compares two images pixel by pixel and returns true, if both are equal for all pixels. As visual result, numerical comparison also showed equality of results.

Despite visual and numerical results proved to be the same, the green area of three NDVI were calculated. An automatic binarization by [15] method was performed, since the NDVI was able to separate vegetation from others targets of the scene. The binarized images are shown in Figure 5, where dark areas do not correspond to the areas of interest, so the calculation was based on bright areas. Based on the binarized images, the green area values obtained for the three NDVI results are shown in Table 1.

As can see by inspecting results, it was found exactly green area for the three NDVI output. It was therefore possible to verify that results with the $c m N D V I$ operator were appropriate and coherent. It is important to bear in mind that the processing was performed in an equivalent form and was used the same data and parameter of gain and offset. In this way, values achieved by the three software could be mathematically compared and shown to be identically, which confirms

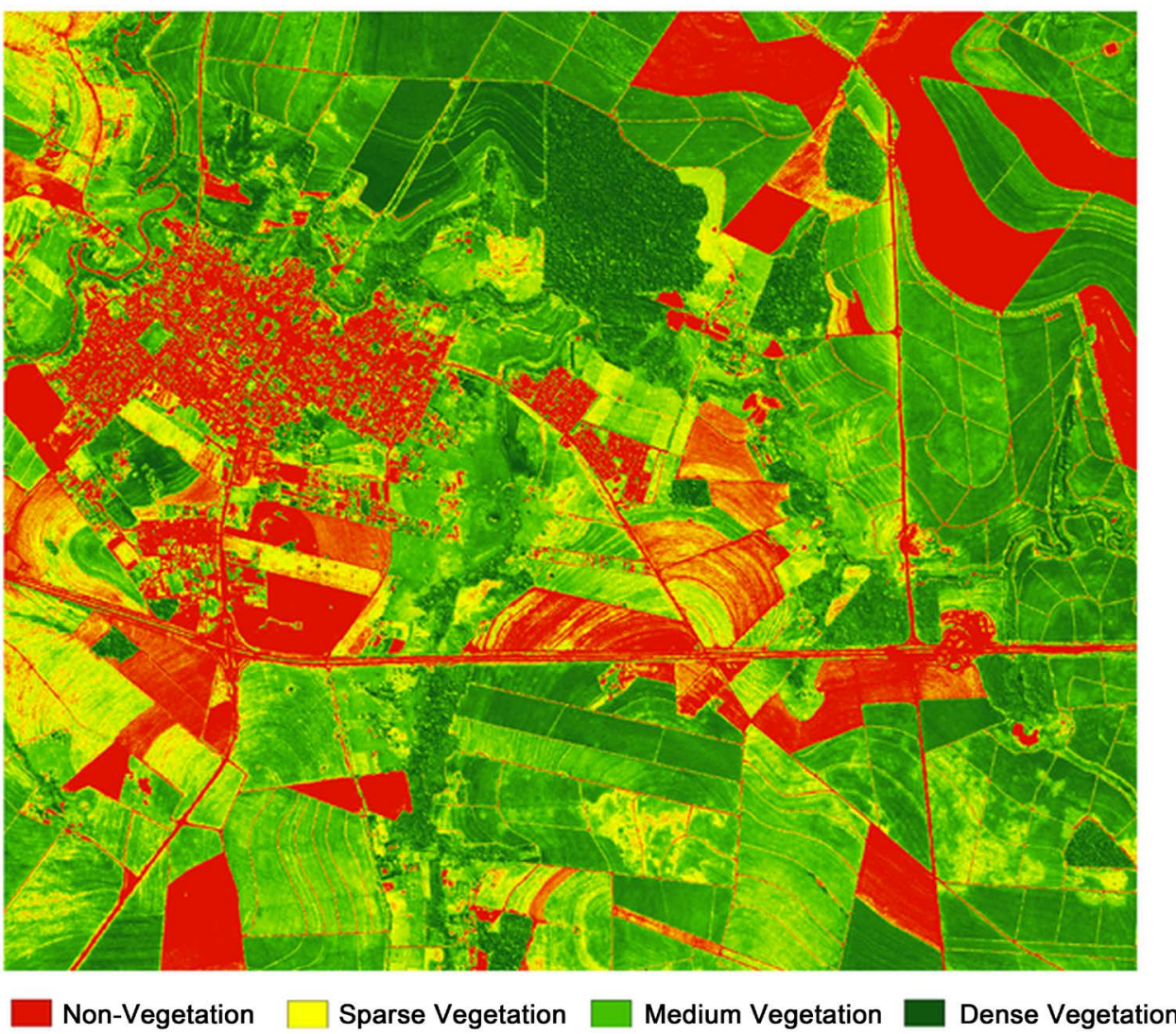

Figure 4. Results from $c m N D V I$ in image's color map with purple being non-vegetated areas, and the darker green representing the more dense vegetated areas. 


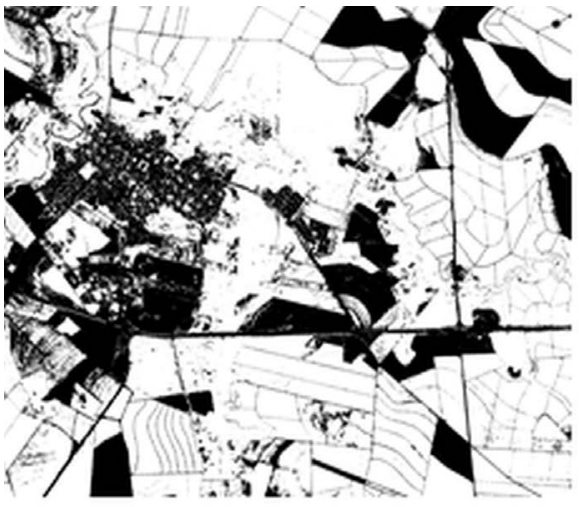

(a)

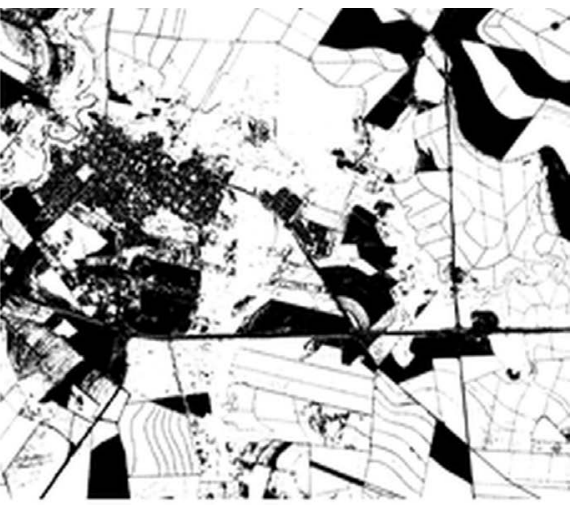

(b)

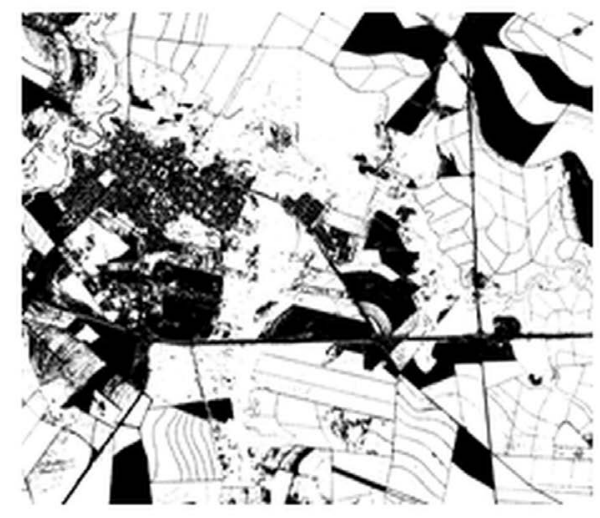

(c)

Figure 5. Binary images from automatic thresholding process for green area calculation, where in black are the excluded pixels that do not pose interesting areas: (a) CARTOMORPH results; (b) SPRING results and (c) ENVI results.

Table 1. Comparison of green area estimation for CARTOMORPH, SPRING and ENVI software.

\begin{tabular}{cc}
\hline NDVI Results & Green Area (pixels) \\
\hline CARTOMORPH & $3,656,822$ \\
SPRING & $3,656,822$ \\
ENVI & $3,656,822$ \\
\hline
\end{tabular}

the consistent output and correctness of the cmNDVI algorithm.

\section{Conclusions}

The evaluation of results achieved with the cmNDVI algorithm available in the CARTOMORPH software indicated a successful implementation. It was noted that vegetation areas showed an expected behaviour, in accordance with the literature, thus being brighter than the other surface targets after the cmNDVI was performed. The output images were visually identical, which can be seen from comparison between the color map and the false color composite of original image. 
We also carried out mathematical evaluation of data, in order to confirm the analyses and results obtained. This evaluation consisted of comparing the outcome from the cmNDVI algorithm available in the CARTOMORPH software and the output from the SPRING and ENVI software. It was found no discrepancies between the three NDVI results, which indicates that the multispectral module of CARTOMORPH software is ready and consistently for operation by users. In addition, the processing of high spectral resolution images should be overcome by CARTOMORPH, since this data provides finer representation within class and it is available nowadays and has been widely employed.

Finally, the importance of vegetation indices, especially NDVI dealt in this study, is already more consolidated and has shown to be a good indicator of vegetation growth and gives some responses to climate change. Therefore, with freely availability of the multispectral module in CARTOMORPH, new applications may be proposed, allowing quantitatively and qualitatively assessing of forest resources distribution, which will be suitable for cartographic applications and to various other fields.

\section{Acknowledgements}

The authors would like to thank FAPESP (Proc. No. 2013/10487-4 and 2013/ 25257-4) for the financial support given to the development of this work.

\section{References}

[1] Mather, P.M. (2004) Computer Processing of Remotely-Sensed Images: An Introduction. 3rd Edition, John Wiley \& Sons, Chichester, $442 \mathrm{p}$.

[2] Poursanidis, D. and Chrysoulakis, N. (2017) Remote Sensing, Natural Hazards and the Contribution of ESA Sentinels Missions. Remote Sensing Applications: Society and Environment, 6, 25-38. https://doi.org/10.1016/j.rsase.2017.02.001

[3] Schultz, M.G., Clevers, J.G.P.W., Carter, S., Verbesselt, J., Avitabilea, V., Quang, H.V. and Herold, M. (2016) Performance of Vegetation Indices from Landsat Time Series in Deforestation Monitoring. International Journal of Applied Earth Observation and Geoinformation, 52, 318-327. https://doi.org/10.1016/j.jag.2016.06.020

[4] Plummer, S., Lecomte, P. and Doherty, M. (2017) The ESA Climate Change Initiative (CCI): A European Contribution to the Generation of the Global Climate Observing System. Remote Sensing of Environment, 203, 2-8. https://doi.org/10.1016/j.rse.2017.07.014

[5] Zhong, Y., Ma, A., Ong, Y.S. and Zhu, Z. (2018) Computational Intelligence in Optical Remote Sensing Image Processing. Applied Soft Computing, 64, 75-93. https://doi.org/10.1016/j.asoc.2017.11.045

[6] Cardim, G.P. (2105) Desenvolvimento do sistema computacional CARTOMORPH para processamento de imagens de sensoriamento remoto. Dissertação (mestrado)-UNESP, Presidente Prudente, 119 p.

[7] Schowengerdt, R.A. (2007) Remote Sensing: Models and Methods for Image Processing. Academic Press, 560 p.

[8] Roy, D.P., Kovalskyy, V., Zhang, H.K., Vermote, E.F., Yan, L., Kumar, S.S. and Egorov, A. (2016) Characterization of Landsat-7 to Landsat-8 Reflective Wavelength and Normalized Difference Vegetation Index Continuity. Remote Sensing of 
Environment, 185, 57-70. https://doi.org/10.1016/j.rse.2015.12.024

[9] Sonawane, K.R. and Bhagat, V.S. (2017) Improved Change Detection of Forests Using Landsat TM and ETM+ Data. Remote Sensing Land, 1, 18-40. https://doi.org/10.21523/gcj1.17010102

[10] Jensen, J.R. (2004) Introductory Digital Image Processing-A Remote Sensing Perspective. 3rd Edition, Prentice Hall, Upper Saddle River, 544 p.

[11] Xie, Y., Sha, Z. and Yu, M. (2008) Remote Sensing Imagery in Vegetation Mapping: A Review. Journal of Plant Ecology, 1, 9-23. https://doi.org/10.1093/jpe/rtm005

[12] Yin, H., Udelhoven, T., Fensholt, R., Pflugmacher, D. and Hostert, P. (2012) How Normalized Difference Vegetation Index (NDVI) Trends from Advanced Very High Resolution Radiometer (AVHRR) and Système Probatoire d'Observation de la Terre VEGETATION (SPOT VGT) Time Series Differ in Agricultural Areas: An Inner Mongolian Case Study. Remote Sensing, 4, 3364-3389. https://doi.org/10.3390/rs4113364

[13] Addabbo, P., Focareta, M., Marcuccio, S., Votto, C. and Ullo, S.L. (2016) Contribution of Sentinel-2 Data for Applications in Vegetation Monitoring. Acta IMEKO, 5 , 44-54. https://doi.org/10.21014/acta_imeko.v5i2.352

[14] Ke, Y., Im, J., Lee, J., Going, H. and Ryu, Y. (2015) Characteristics of Landsat 8 OLI-Derived NDVI by Comparison with Multiple Satellite Sensors and In-Situ Observations. Remote Sensing of Environment, 164, 298-313. https://doi.org/10.1016/j.rse.2015.04.004

[15] Otsu, N. (1979) A Threshold Selection Method from Gray-Level Histograms. IEEE Transactions on Systems, Man and Cybernetics, 9, 62-69.

https://doi.org/10.1109/TSMC.1979.4310076 